\title{
Cervical pessary for preterm twin pregnancy in women with a short cervix
}

\author{
Da Un Jung, MD, Min Jung Choi, MD, Sun Young Jung, MD, Suk Young Kim, MD, PhD \\ Department of Obstetrics and Gynecology, Gachon University Gil Medical Center, Incheon, Korea
}

Objective

We aimed to evaluate the effectiveness of cervical pessary for extending twin pregnancy in women with a short cervix.

\section{Methods}

Between January 2014 and March 2019, the use of a cervical pessary for twin pregnancy in women with a cervical length of $\leq 15 \mathrm{~mm}$ was investigated between 16 and 28 weeks of gestation. We included women with a shortened cervix after a cerclage procedure and visible fetal membranes and cervical dilatation. Thirteen patients underwent pessary insertion and were matched with a control group of 15 patients. Pregnancy and neonatal outcomes were compared between the groups. We excluded women with major fetal anomalies discovered before or after birth and known placenta previa.

\section{Results}

Women who underwent pessary insertion were diagnosed with a short cervix $(0.65 \pm 0.47 \mathrm{vs} .0 .66 \pm 0.51 \mathrm{~cm}$ in the pessary and control groups, respectively; $P=0.957)$ at a gestational age similar to that of the controls ( 23.29 vs. 25.14 weeks, $P=0.294$ ). There was no significant difference in gestational age at delivery between them (33.29 vs. 27.29 weeks in the pessary and control groups, respectively, $P=0.058)$. The pessary group had a longer interval between the diagnosis and delivery than the control group $(60.7 \pm 28.3$ vs. $22.6 \pm 24.5$ days, $P=0.001)$. The number of adverse neonatal outcomes was significantly lower in the pessary group $(n=7 ; 28.0 \%)$ than in the control group $(n=14 ; 58.3 \%$; $P=0.032$ ).

\section{Conclusion}

The use of a cervical pessary may prolong gestation in twin pregnancies in women with an extremely short cervix until viable gestational age.

Keywords: Twin pregnancy; Cervical length measurement; Pessaries; Preterm birth; Prenatal ultrasonography

\section{Introduction}

Spontaneous preterm birth (SPB) is a major cause of neonatal mortality and morbidity. In 2016, preterm births accounted for $9.85 \%$ of all births in the United States of America. Preterm birth accounted for $59.91 \%$ of all twin births versus $8.02 \%$ of all singleton births [1]. One of the best methods for predicting SPB is the assessment of the cervical length $(\mathrm{CL})$ on second-trimester transvaginal sonography [2-5].

The interventions known to be effective for preventing SPB in singleton pregnancies include bed rest, prophylactic cervical cerclage, and progesterone supplementation $[6,7]$. Unfortunately, none of these interventions is effective for high-risk
Received: 2019.06.12. Revised: 2019.08.22. Accepted: 2019.08.25. Corresponding author: Suk Young Kim, MD, PhD

Department of Obstetrics and Gynecology, Gachon Medical School Gil Medical Center, 21 Namdong-daero 774beon-gil, Namdonggu, Incheon 21565, Korea

E-mail: ksyob@gilhospital.com

https://orcid.org/0000-0003-3600-9691

Articles published in Obstet Gynecol Sci are open-access, distributed under the terms of the Creative Commons Attribution Non-Commercial License (http://creativecommons. org/licenses/by-nc/3.0/) which permits unrestricted non-commercial use, distribution, and reproduction in any medium, provided the original work is properly cited.

Copyright $\odot 2020$ Korean Society of Obstetrics and Gynecology 


\title{
Obstetrics \& Gynecology Science
}

\author{
Vol. 63, No. 3, 2020
}

twin pregnancies [8-10]. Cervical pessaries, which have not been widely used in Korea, have been investigated for their ability to reduce preterm birth rates. For centuries, vaginal pessaries have been used for vaginal prolapse. Many gynecologists have used pessaries due to fitting and management convenience. In 1959, Cross reported using a ring pessary to treat cervical laceration, cervical incompetence, or uterus didelphys [11], and some physicians attempted the use of pessaries for prophylaxis of SPB. In the late 1970s, Hans Arabin designed a dome-shaped, flexible silicone pessary [12]. The Arabin pessary supports the cervix and consequently prevents opening of the internal os, changes the uterocervical angle to shift the uterine weight toward the lower anterior segment rather than directly on the cervix, and protects the cervical mucus plug, which acts as a protective film to prevent infection [13]. Several studies have investigated the effectiveness of cervical pessaries in twin pregnancies; however, the conclusions remain controversial. Our study aimed to evaluate cervical pessaries as an option for preventing SPB in twin pregnancies among women with a short cervix.

\section{Materials and methods}

Women with twin pregnancies at 16-28 weeks of gestation were included. All women underwent transvaginal ultrasonography with an empty bladder. A $C L$ of $\leq 15 \mathrm{~mm}$ was considered short. We reviewed the charts of all patients with a twin pregnancy between January 2014 and March 2019. We included women with a shortened $\mathrm{CL}$ after a cerclage procedure and visible fetal membranes with cervical dilatation. Three of our patients had membrane bulging and one patient in the pessary group underwent amnioreduction of $120 \mathrm{~mL}$ because of protruding membranes. We excluded women with major fetal anomalies discovered before or after birth and known placenta previa. Since 2016, patients were informed of the potential advantages, and pessaries were placed only in those who agreed to participate. The use of a cervical pessary for prophylaxis of SPB is not approved by the Korean Food and Drug Administration. All patients were informed of its use, and written informed consent was obtained prior to insertion. The study was approved by the Institutional Review Board (IRB) and Ethics Committee of Gachon University Gil Medical Center (IRB number GCIRB2018-198) and performed in accordance with the principles of the Dec- laration of Helsinki. All patients were prescribed $200 \mathrm{mg}$ of vaginal progesterone daily. Study patients with a short cervix underwent Arabin pessary placement (ARABIN ${ }^{\circledR}$ Cerclage Pessary by Dr. Arabin, GmbH \& Co. KG, Witten, Germany); controls with a short cervix did not undergo pessary placement. Women in both groups were prescribed antibiotics if the C-reactive protein (CRP) level was higher than normal or when inflammation was suspected. When no signs of inflammation were apparent and the CRP level was acceptable, the pessaries were inserted. A flexible ring-like silicone Arabin pessary was offered in the pessary group, which is inexpensive and can be non-invasively and easily placed and removed without anesthesia [14]. After taking cervical swabs, the operator unfolded and placed a pessary around the cervix, with the small ring toward the cervix and the large ring toward the pelvic floor, and then pressed it slightly toward the sacrum. If the cervical swab detected bacteria, patients were treated with appropriate antibiotics. The pessary was sized according to the instructions provided by Arabin and Alfirevic after measuring the cervix on transvaginal sonography and performing pelvic examination using a speculum [14]. Pessary removal should be performed before the onset of delivery or at around 37 weeks. The pessary can be removed easily without the use of any instrument. Arabin and Alfirevic advised pushing the cervix back through the inner ring of the pessary [14]. In our study, the pessary was removed at 36 weeks in one patient prior to vaginal delivery and immediately before or after cesarean section in the other patients. In all treated patients, CL was measured via transvaginal sonography before and after pessary insertion. Fig. 1 shows the sonographs after pessary insertion according to the new transvaginal technique provided by Goya et al. [15], in which the transvaginal probe is inserted to the pessary to touch the anterior cervical lip. Continuous variables were compared using Student's t-test or the Mann-Whitney U-test. Categorical variables were evaluated using Pearson's $\chi^{2}$ test or Fisher's exact test. The risk of preterm birth was quantified as odds ratios and 95\% confidence intervals ( $\mathrm{Cls}$ ) using logistic regression. The probability of continued pregnancy was assessed using Kaplan-Meier analysis (Fig. 2), in which delivery at $<34$ weeks was the outcome. IBM ${ }^{\circledast}$ SPSS ${ }^{\circledR}$ Statistics version 22 (SPSS Inc., Chicago, IL, USA) was used for the statistical analysis, and $P<0.05$ were considered significant. 


\section{Obstetrics \& Gynecology Science}

Da Un Jung, et al. Pessary for twin with a short cervix

\section{Results}

Nineteen women were initially included in the study. Six were excluded from the analysis because of loss to followup or insufficient data. Therefore, 13 women with a $C L$ of $\leq 15 \mathrm{~mm}$ underwent pessary placement and 15 women with a CL of $\leq 15 \mathrm{~mm}$ were considered controls and did not receive pessary placement. Baseline $C L$ values $(0.65 \pm 0.47$ vs. $0.66 \pm 0.51 \mathrm{~cm}$ in the treatment and control groups, respectively; $P=0.957$ ) and gestational ages ( 23.29 vs. 25.14 weeks; $P=0.294$ ) were similar between the groups. The other characteristics are described in Table 1. There was no significant preterm history in either group. Three women in the pessary group and four in the control group were multigravidas. One patient in each group underwent a biopsy using a loop electrosurgical excision procedure (LEEP). Of the six patients who received a cerclage in the pessary group, two underwent the procedure at other clinics. Of the five patients who received cerclage in the control group, three underwent the procedure at a local clinic. Four patients in the pessary group and two in the control group received rescue cerclage in our hospital prior to the pessary insertion. Dichorionic twins
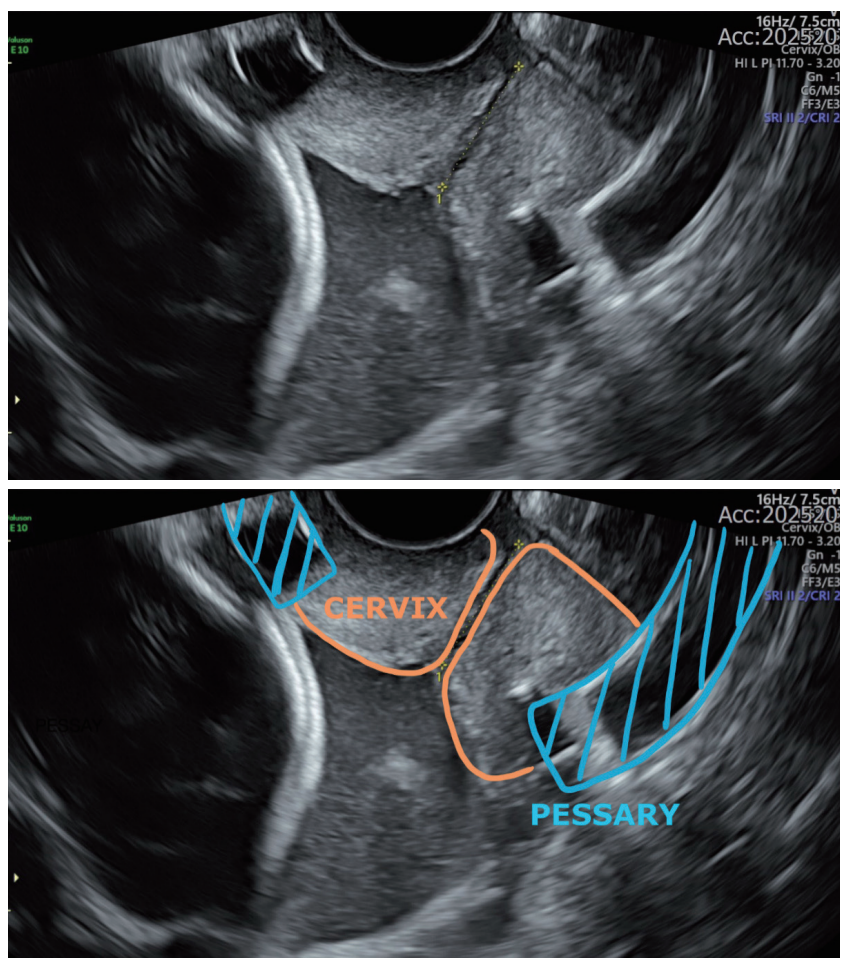

Fig. 1. Transvaginal sonographs showing cervical length after pessary insertion. were more common in the pessary group than in the control group because three-fourth of women in the pessary group became pregnant through in vitro fertilization (IVF). There was no significant difference in the initial hemoglobin level, white blood cell count, or CRP level between the two groups. The mean $C L$ after pessary insertion increased by approximately $1.68 \pm 0.78 \mathrm{~cm}$. Pessary insertion was performed at a median gestational age of 24.29 weeks. In some women, it took several days to prepare for pessary insertion. The length of hospital stays ranged from 2 to 97 days for patients who received pessary placement, with a median of 11 days.

Pregnancy outcomes are shown in Table 2 . The median gestational age at delivery in the pessary group was higher than that in the control group (33.29 vs. 27.29 weeks, respectively; $P=0.058$ ). One woman who underwent pessary placement had a term birth at 37.4 weeks of gestation; however, none of the patients in the control group had given birth at $\geq 37$ weeks. A cervical pessary can help significantly prolong the time to delivery $(60.69 \pm 28.26$ vs. $22.60 \pm 24.45$ days; $P=0.001$ ). Analysis of covariance controlling for the difference at baseline (IVF and chorionicity) showed that the mean gestational age at delivery was $32.07 \pm 1.46$ weeks in the pessary group and $27.16 \pm 1.34$ weeks in the control group (adjusted $P=0.032$ ) and that the time from random-

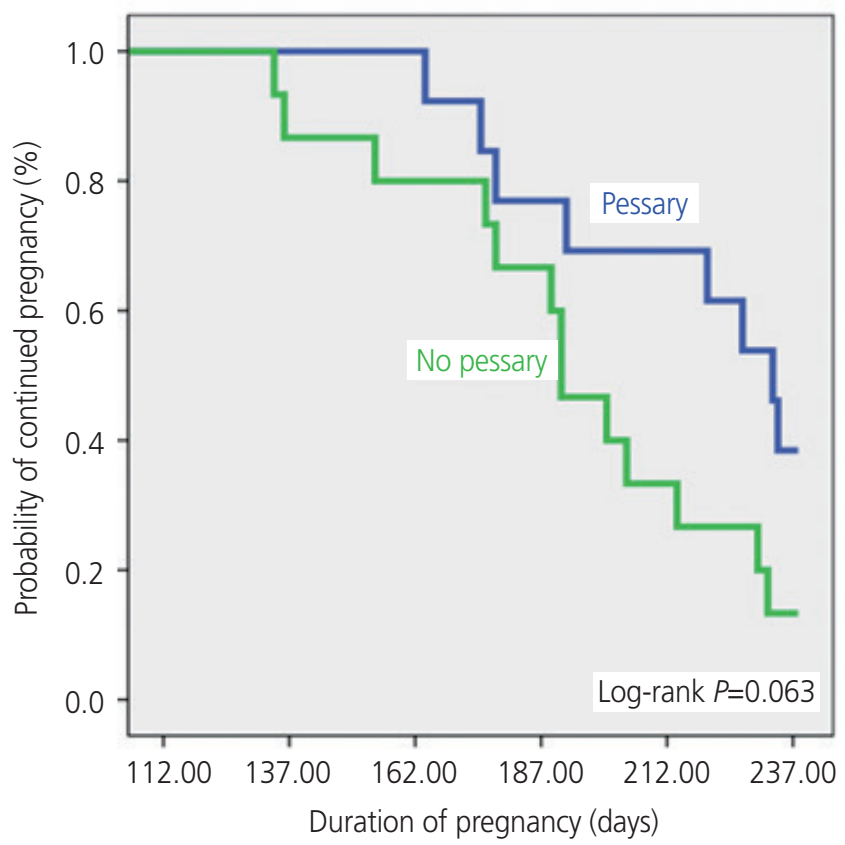

Fig. 2. Kaplan-Meier curves of probability of continued pregnancy. 


\section{Obstetrics \& Gynecology Science}

Vol. 63 , No. 3, 2020

ization to delivery was significantly different between the groups ( $60.38 \pm 8.4$ vs. $22.87 \pm 7.7$ days; adjusted $P=0.006$ ). The use of a cervical pessary tended to reduce preterm birth at $<32$ weeks; however, the difference was not significant (38.5\% vs. $73.3 \%$, odds ratio, $0.227 ; 95 \% \mathrm{Cl}, 0.046-1.125$; $P=0.069$ ). Fig. 1 shows the Kaplan-Meier analysis of the cumulative percentage of patients who did not give birth before 34 weeks, where gestational age was the time scale and all pregnancies were considered no longer at risk for any event at the start of the 34th week. The probability of continued pregnancy did not differ between the groups (log-rank, $P=0.063$ ).

One woman in the pessary group had a stillbirth at 23.4 weeks, and six in the control group had a stillbirth before 22 weeks. The use of a cervical pessary was associated with a higher birth weight, particularly fewer neonates with a birth weight of $<1,500$ or $<1,000 \mathrm{~g}$ (Table 3).

Neonatal intensive care unit admission was required for

Table 1. Characteristics of the study participants

\begin{tabular}{|c|c|c|c|}
\hline Characteristic & Pessary $(n=13)$ & Control $(n=15)$ & $P$-value \\
\hline Age (yr) & $32.92 \pm 2.78$ & $32.80 \pm 2.40$ & 0.641 \\
\hline Height (cm) & $161.54 \pm 5.64$ & $161.67 \pm 6.26$ & 0.955 \\
\hline Weight (kg) & $68.77 \pm 13.40$ & $65.20 \pm 10.69$ & 0.440 \\
\hline $\mathrm{BMI}\left(\mathrm{kg} / \mathrm{m}^{2}\right)$ & $26.24 \pm 4.21$ & $24.88 \pm 3.38$ & 0.352 \\
\hline IVF & $10(76.9)$ & $5(33.3)$ & 0.030 \\
\hline Cerclage op & $6(46.2)$ & $5(33.3)$ & 0.488 \\
\hline \multicolumn{4}{|l|}{ Chorionicity } \\
\hline Dichorionic & $13(100.0)$ & $8(53.3)$ & 0.007 \\
\hline Monochorionic & $0(0.0)$ & $7(46.7)$ & 0.007 \\
\hline Initial CRP (mg/dL) & $0.91 \pm 1.60$ & $1.18 \pm 1.60$ & 0.369 \\
\hline Initial Hb (g/dL) & $10.97 \pm 1.29$ & $11.07 \pm 1.33$ & 0.836 \\
\hline Initial WBC (/mm³) & $10,860.77 \pm 3,032.21$ & $10,376.67 \pm 2,236.40$ & 0.632 \\
\hline Gestational age at randomization (wk) & $23.29(20.5-26.0)$ & $25.14(21.9-26.4)$ & 0.294 \\
\hline Initial cervical length $(\mathrm{cm})$ & $0.65 \pm 0.47$ & $0.66 \pm 0.51$ & 0.957 \\
\hline Cervical length after pessary insertion $(\mathrm{cm})$ & $1.68 \pm 0.78$ & - & \\
\hline Gestational age at pessary insertion (wk) & $24.29(22.1-26.1)$ & - & \\
\hline Admission days after pessary insertion (day) & $11(4-25.5)$ & - & \\
\hline
\end{tabular}

Data are presented as numbers (percentages), means \pm standard deviations, or medians (interquartile ranges).

IVF, in vitro fertilization; CRP, C-reactive protein; $\mathrm{Hb}$, hemoglobin; WBC, white blood cell.

Table 2. Pregnancy outcomes

\begin{tabular}{|c|c|c|c|c|}
\hline Pregnancy outcome & Pessary $(n=13)$ & Control $(n=15)$ & Odds ratio $(95 \% \mathrm{Cl})$ & $P$-value \\
\hline Gestational age at delivery (wk) & $33.29(26.4-34.8)$ & $27.29(25.1-32.9)$ & - & 0.058 \\
\hline Days to delivery (day) & $60.69 \pm 28.26$ & $22.60 \pm 24.45$ & - & 0.001 \\
\hline \multicolumn{5}{|l|}{ Preterm birth } \\
\hline$>24$ wk of gestation & $1(7.7)$ & $3(20.0)$ & $0.333(0.030-3.676)$ & 0.370 \\
\hline$>28 w k$ of gestation & $4(30.8)$ & $8(53.3)$ & $0.389(0.082-1.840)$ & 0.234 \\
\hline$>32$ wk of gestation & $5(38.5)$ & $11(73.3)$ & $0.227(0.046-1.125)$ & 0.069 \\
\hline >34 wk of gestation & $8(61.5)$ & $13(86.7)$ & $0.246(0.038-1.583)$ & 0.140 \\
\hline
\end{tabular}

Data are presented as numbers (percentages), means \pm standard deviations, or medians (interquartile ranges).

$\mathrm{Cl}$, confidence interval. 


\section{Obstetrics \& Gynecology Science}

Da Un Jung, et al. Pessary for twin with a short cervix

$84 \%$ of neonates in the pessary group versus all of those in the control group. The rates of adverse neonatal outcomes in the pessary group were significantly different from those in the control group (Table 4).

\section{Discussion}

In our study, the use of a cervical pessary to shorten the cervix to $<15 \mathrm{~mm}$ was associated with prolongation of a twin pregnancy. This suggests that its use can effectively postpone delivery until a viable gestational age, especially in twin pregnancies. Two large randomized controlled trials (RCTs) examined the use of a cervical pessary in singleton pregnancy. The PECEP trial compared a pessary group $(n=192)$ and an expectant management group $(n=193)$ with a $C L$ of $\leq 25 \mathrm{~mm}$. The rate of spontaneous delivery before 34 weeks of gestation was significantly lower in the pessary group $(n=12 ; 6 \%)$ than in the expectant management group ( $n=51$; 27\%; $P<0.001$ ) [16]. However, a multinational trial observed no significant difference in the rate of spontaneous early preterm delivery before 34 weeks between 465 women who underwent pessary placement $(n=55 ; 12.0 \%)$ and 467 who did not ( $n=50 ; 10.8 \% ; P=0.57$ ) [17]. The use of a cervical pessary in twin pregnancy was studied in three RCTs [18-20], of which two demonstrated that its use was not associated with a reduction in preterm delivery among unselected twin pregnancies. In the ProTwin trial, the subgroup analysis for those with a $\mathrm{CL}$ of $<38 \mathrm{~mm}$ showed that the use of a pessary reduced the risk of preterm delivery before 28 weeks $(n=3 ; 4 \%$, in those who underwent pessary placement vs. $\mathrm{n}=9 ; 16 \%$, in those who did not; $P=0.0158$ ) or 32 weeks

Table 3. Perinatal outcomes

\begin{tabular}{lccc}
\hline Perinatal outcome & Pessary $(\mathbf{n}=\mathbf{2 6})$ & Control $(\mathbf{n}=\mathbf{3 0})$ & $\boldsymbol{P}$-value \\
\hline Birth weight $(\mathrm{g})$ & $1,687.88 \pm 731.91$ & $1,074.67 \pm 625.52$ & 0.001 \\
$<1,000$ & $6(23.1)$ & $17(56.7)$ & 0.011 \\
$<1,500$ & $8(30.8)$ & $24(80)$ & 0.000 \\
$<2,500$ & $23(88.5)$ & $30(100)$ & 0.094 \\
Fetal death & $1(3.8)$ & $6(20)$ & 0.108 \\
\hline
\end{tabular}

Data are presented as numbers (percentages) or means \pm standard deviations.

Table 4. Neonatal outcomes

\begin{tabular}{lccc}
\hline Neonatal outcome & Pessary $(\mathbf{n}=\mathbf{2 5})$ & Control $(\mathbf{n = 2 4 )}$ & $\boldsymbol{P}$-value \\
\hline 1-min Apgar less than 7 & $5(20.0)$ & $7(29.2)$ & 0.456 \\
5-min Apgar less than 7 & $2(8.0)$ & $2(8.3)$ & 0.999 \\
NICU admission & $21(84)$ & $24(100)$ & 0.110 \\
NICU admission (day) & $33.88 \pm 38.61$ & $49.83 \pm 39.82$ & 0.089 \\
Adverse neonatal outcome & $7(28.0)$ & $14(58.3)$ & 0.032 \\
Death & $1(4.0)$ & $2(51.1)$ & 0.609 \\
Respiratory distress syndrome & $6(24.0)$ & $11(45.8)$ & 0.108 \\
Intraventricular hemorrhage & $1(4.0)$ & $3(12.5)$ & 0.349 \\
Patent ductus arteriosis & $1(4.0)$ & $3(12.5)$ & 0.349 \\
Retinopathy of prematurity & $4(16.0)$ & $4(16.7)$ & 0.999 \\
Necrotizing enterocolitis & $4(16.0)$ & $4(16.7)$ & 0.999 \\
Sepsis & $0(0.0)$ & $3(12.5)$ & 0.110 \\
Mechanical ventilation & $7(28.0)$ & $14(58.3)$ & 0.032 \\
\hline
\end{tabular}

Data are presented as numbers (percentages) or means \pm standard deviations.

NICU, neonatal intensive care unit. 


\section{Obstetrics \& Gynecology Science}

Vol. 63, No. 3, 2020

( $n=11 ; 14 \%$ vs. $n=16 ; 29 \% ; P=0.0476)$ [20]. The PECEPTwins trial showed that a cervical pessary prevented preterm birth before 34 weeks in women with a twin pregnancy and a short cervix (CL of $<25 \mathrm{~mm}$; pessary group, 12/68 [17.6\%] vs. control group, $27 / 66$ [40.9\%]; $P=0.002$ ) [18]. Although a cervical pessary did not reduce the rate of preterm delivery in unselected twin pregnancies, these studies showed the potential value of prophylaxis against SPB in women with twin pregnancies and a very short cervix. Therefore, we began to investigate whether the use of a cervical pessary would help protect against SPB in twin pregnancies, similar to that with the use of cerclage or progesterone administration. Moreover, we found no study on the use of a cervical pessary in twin pregnancies in Korea. Unlike in previous trials, we selected and investigated women with twin pregnancies and an extremely short cervix ( $\leq 15 \mathrm{~mm}$ ). We focused on patients at a high risk of SPB who needed immediate intervention. Hassan et al. reported that patients with a $C L$ of $\leq 15 \mathrm{~mm}$ needed urgent intervention because of a $50 \%$ risk of SPB [21]. This study did not show that the use of a cervical pessary significantly reduced the rate of SPB compared with the controls but showed that its use could help prolong a pregnancy until viable gestational age in women with impending childbirth. The Arabin pessary is inexpensive, is relatively safe, and has few side effects. Half of the patients in the pessary group reported increased vaginal discharge; however, none requested pessary removal because of discomfort. Arabin et al. [13] reported that contraindications for the use of a pessary included lethal fetal abnormality, suspected chorioamnionitis, ballooning of membranes into the vagina, effective uterine contractions, and uterine anomalies, such as the presence of two cervices.

Although the gestational age at birth did not differ significantly between the groups, the birth weight in the pessary group tended to be higher than that in the control group. The use of a pessary reduced the rate of adverse neonatal outcomes and need for mechanical ventilation.

Our study had some strengths. All participants had a very short cervix ( $C L$ of $\leq 15 \mathrm{~mm}$ ), and the same doctor performed all procedures, eliminating all procedure-related bias. In contrast with Europe, in Korea, cervical pessaries are not widely used to prevent SPB in women with twin pregnancies and a short cervix [22]. Our study may encourage further research on the use of a cervical pessary in Korea and other Asian countries. As limitations, this small retrospective study was performed in a medical center and did not consider cervical cerclage, IVF, chorionicity, or other unknown factors that might have influenced the outcomes. Our results raise several concerns: if pessaries can be used for prophylaxis after cervical conization or LEEP, if progesterone supplementation is effective after pessary insertion, and when outpatient follow-up should be performed after pessary insertion. Further research is needed to address these concerns in addition to ongoing research on the role of prior cerclage.

No reported interventions, to date, were effective in twin pregnancy $[9,23]$ in contrast with singleton pregnancy; thus, it is necessary to consider alternative methods. The use of a cervical pessary may reduce the rates of SPB and adverse neonatal outcomes in twin pregnancy. When it is necessary to prolong gestation in a pre-viable twin pregnancy, a cervical pessary may be used. Thus, research on the use of a cervical pessary should continue.

In conclusion, the use of a cervical pessary may prolong gestation in women with twin pregnancy and an extremely short cervix until viable gestational age. The pessary group had fewer adverse neonatal outcomes than the control group. However, further large and well-designed trials are needed to verify our findings.

\section{Conflict of interest}

No potential conflict of interest relevant to this article was reported.

\section{Ethical approval}

The study was approved by the IRB of Gachon University Gil Medical Center (IRB no. GCIRB2018-198) and performed in accordance with the principles of the Declaration of Helsinki.

\section{Patient consent}

Written informed consent was obtained from each participant. 


\section{Obstetrics \& Gynecology Science}

Da Un Jung, et al. Pessary for twin with a short cervix

\section{References}

1. Martin JA, Hamilton BE, Osterman MJK, Driscoll AK, Drake P. Births: final data for 2016. Natl Vital Stat Rep 2018;67:1-55.

2. Ehsanipoor RM, Haydon ML, Lyons Gaffaney C, Jolley $J A$, Petersen R, Lagrew DC, et al. Gestational age at cervical length measurement and preterm birth in twins. Ultrasound Obstet Gynecol 2012;40:81-6.

3. Fox NS, Saltzman DH, Fishman A, Klauser CK, Gupta S, Rebarber A. Gestational age at cervical length and fetal fibronectin assessment and the incidence of spontaneous preterm birth in twins. J Ultrasound Med 2015;34:977-84.

4. Goldenberg RL, lams JD, Miodovnik M, Van Dorsten JP, Thurnau $G$, Bottoms $S$, et al. The preterm prediction study: risk factors in twin gestations. Am J Obstet Gynecol 1996;175:1047-53.

5. Lim AC, Hegeman MA, Huis In 'T Veld MA, Opmeer BC, Bruinse HW, Mol BW. Cervical length measurement for the prediction of preterm birth in multiple pregnancies: a systematic review and bivariate meta-analysis. Ultrasound Obstet Gynecol 2011;38:10-7.

6. Fonseca EB, Celik E, Parra M, Singh M, Nicolaides KH; Fetal Medicine Foundation Second Trimester Screening Group. Progesterone and the risk of preterm birth among women with a short cervix. N Engl J Med 2007;357:462-9.

7. Hassan SS, Romero R, Vidyadhari D, Fusey S, Baxter $J K$, Khandelwal $M$, et al. Vaginal progesterone reduces the rate of preterm birth in women with a sonographic short cervix: a multicenter, randomized, double-blind, placebo-controlled trial. Ultrasound Obstet Gynecol 2011;38:18-31.

8. Crowther CA, Han S. Hospitalisation and bed rest for multiple pregnancy. Cochrane Database Syst Rev 2010;(7):CD000110.

9. Roman A, Rochelson B, Fox NS, Hoffman M, Berghella V, Patel $V$, et al. Efficacy of ultrasound-indicated cerclage in twin pregnancies. Am J Obstet Gynecol 2015;212:788. e1-6.

10. Norman JE, Mackenzie F, Owen P, Mactier H, Hanretty K, Cooper $S$, et al. Progesterone for the prevention of preterm birth in twin pregnancy (STOPPIT): a randomised, double-blind, placebo-controlled study and meta-analy- sis. Lancet 2009;373:2034-40.

11. Cross RG. Treatment of habitual abortion due to cervical incompetence. Lancet 1959;274:127.

12. Arabin H. Pessartherapie (therapy with pessaries). New York (NY): Thieme; 1991. p. 263-76.

13. Arabin B, Halbesma JR, Vork F, Hübener M, van Eyck J. Is treatment with vaginal pessaries an option in patients with a sonographically detected short cervix? J Perinat Med 2003;31:122-33.

14. Arabin B, Alfirevic Z. Cervical pessaries for prevention of spontaneous preterm birth: past, present and future. Ultrasound Obstet Gynecol 2013;42:390-9.

15. Goya M, Pratcorona L, Higueras T, Perez-Hoyos S, Carreras $E$, Cabero $L$. Sonographic cervical length measurement in pregnant women with a cervical pessary. Ultrasound Obstet Gynecol 2011;38:205-9.

16. Goya M, Pratcorona L, Merced C, Rodó C, Valle L, Romero $A$, et al. Cervical pessary in pregnant women with a short cervix (PECEP): an open-label randomised controlled trial. Lancet 2012;379:1800-6.

17. Nicolaides KH, Syngelaki A, Poon LC, Picciarelli G, Tul $\mathrm{N}$, Zamprakou A, et al. A randomized trial of a cervical pessary to prevent preterm singleton birth. N Engl J Med 2016;374:1044-52.

18. Goya M, de la Calle M, Pratcorona L, Merced C, Rodó C, Muñoz B, et al. Cervical pessary to prevent preterm birth in women with twin gestation and sonographic short cervix: a multicenter randomized controlled trial (PECEPTwins). Am J Obstet Gynecol 2016;214:145-52.

19. Nicolaides KH, Syngelaki A, Poon LC, de Paco Matallana C, Plasencia W, Molina FS, et al. Cervical pessary placement for prevention of preterm birth in unselected twin pregnancies: a randomized controlled trial. Am J Obstet Gynecol 2016;214:3.e1-9.

20. Liem S, Schuit E, Hegeman M, Bais J, de Boer K, Bloemenkamp K, et al. Cervical pessaries for prevention of preterm birth in women with a multiple pregnancy (ProTWIN): a multicentre, open-label randomised controlled trial. Lancet 2013;382:1341-9.

21. Hassan SS, Romero R, Berry SM, Dang K, Blackwell SC, Treadwell MC, et al. Patients with an ultrasonographic cervical length $\leq 15 \mathrm{~mm}$ have nearly a $50 \%$ risk of early spontaneous preterm delivery. Am J Obstet Gynecol 2000;182:1458-67.

22. Dharan VB, Ludmir J. Alternative treatment for a 


\section{Obstetrics \& Gynecology Science}

Vol. 63, No. 3, 2020

short cervix: the cervical pessary. Semin Perinatol 2009;33:338-42.

23. Saccone G, Rust O, Althuisius S, Roman A, Berghella V. Cerclage for short cervix in twin pregnancies: systematic review and meta-analysis of randomized trials using individual patient-level data. Acta Obstet Gynecol Scand 2015;94:352-8. 